\title{
Yttrium-90 resin microspheres as an adjunct to sorafenib in patients with unresectable hepatocellular carcinoma
}

This article was published in the following Dove Press journal:

Journal of Hepatocellular Carcinoma

5 February 2016

Number of times this article has been viewed

\author{
Armeen Mahvash' \\ Ravi Murthy' \\ Bruno C Odisio' \\ Kanwal Pratap Raghav ${ }^{2}$ \\ Lauren Girard ${ }^{2}$ \\ Sheree Cheung' \\ Van Nguyen ${ }^{3}$ \\ Joe Ensor ${ }^{4}$ \\ Sameer Gadani ${ }^{5}$ \\ Khaled M Elsayes ${ }^{6}$ \\ Reham Abdel-Wahab ${ }^{1,7}$ \\ Manal Hassan' \\ Ahmed S Shalaby' \\ James CYao' \\ Michael J Wallace' \\ Ahmed O Kaseb ${ }^{2}$
}

'Department of Interventional Radiology, ${ }^{2}$ Department of Gastrointestinal Medical Oncology, ${ }^{3}$ Department of Pharmacy, ${ }^{4}$ Department of Statistics, The University of Texas MD Anderson Cancer Center, Houston, TX, ${ }^{5}$ Department of Radiology, Saint Louis University Hospital, St Louis, MO, ' Department of Diagnostic Radiology, The University of Texas MD Anderson Cancer Center, Houston, TX, USA;

${ }^{7}$ Department of Clinical Oncology, Assiut University Hospital, Assiut, Egypt

Correspondence: Armeen Mahvash Department of Interventional Radiology, Unit 147I, The University of Texas MD Anderson Cancer Center, I5I5 Holcombe Boulevard, Houston, TX 77030, USA

Tel + I 7135637340

Fax + I 7|3745 II63

Email amahvash@mdanderson.org

Ahmed O Kaseb

Department of Gastrointestinal Medical Oncology, Unit 426, The University of Texas MD Anderson Cancer Center, 1515 Holcombe Boulevard, Houston, TX 77030, USA

Tel +l 7137922828

Fax + I 7/3745 II63

Email akaseb@mdanderson.org
Purpose: The safety and efficacy of the combined use of sorafenib and yttrium-90 resin microspheres (Y90 RMS) to treat advanced hepatocellular carcinoma (HCC) is not well established. We determined the incidence of adverse events with this combination therapy in patients with advanced HCC at our institution and analyzed the treatment and survival outcomes.

Materials and methods: We reviewed the records of 19 patients with Barcelona Clinic Liver Cancer class B or C HCC who underwent treatment with Y90 RMS (for 21 sessions) while receiving full or reduced doses of sorafenib between January 2008 and May 2010. Therapy response was evaluated using Response Evaluation Criteria in Solid Tumors. We evaluated median overall survival (OS) and progression-free survival (PFS) as well as hepatic and extrahepatic disease PFS and incidence of adverse events.

Results: The median patient age was 67 years, and portal or hepatic venous invasion was present in eight patients (42\%). Ten patients received reduced doses of sorafenib. The median Y90 radiation activity delivered was $41.2 \mathrm{mCi}$. The partial response of Response Evaluation Criteria in Solid Tumors was observed in four patients (19\%). The median hepatic disease PFS was 7.82 months, extrahepatic disease PFS was 8.94 months, OS was 19.52 months, and PFS was 6.63 months. Ninety days after treatment with Y90 RMS, five patients (26\%) had grade II adverse events and four patients (21\%) had grade III adverse events.

Conclusion: OS and PFS outcomes were superior to those observed in prior studies evaluating sorafenib alone in patients with a similar disease status, warranting further study of this treatment combination.

Keywords: yttrium-90, sorafenib, hepatocellular carcinoma

\section{Introduction}

Hepatocellular carcinoma (HCC) is the fifth most common cancer and third leading cause of cancer-related deaths worldwide. ${ }^{1}$ When the disease is diagnosed at early stages, it is amenable to potentially curative treatments, such as surgical therapies (resection or liver transplantation) and locoregional procedures (percutaneous thermal ablation). Five-year survival rates of up to $60 \%-70 \%$ can be achieved in well-selected patients. ${ }^{2}$ However, when HCC is diagnosed at an advanced stage, prognosis is poor owing to a combination of underlying liver disease and lack of effective treatment options. ${ }^{1-3}$ The most common cause of death in patients with advanced HCC is liver failure due to local disease progression, and survival rates quickly decline in these patients: the 1-year survival rate is $29 \%$, the 3 -year survival rate is $8 \%$, and the 5 -year survival rate is $0 \%{ }^{4}$

The efficacy and safety of sorafenib for the treatment of HCC has been well established in multiple multinational Phase III trials (Sorafenib HCC Assessment submit your manuscript | www.dovepress.com

Dovepress

http://dx.doi.org//0.2147/JHC.S62261
Journal of Hepatocellular Carcinoma 2016:3 1-7

(c) (i) (5) 2016 Mahvash et al. This work is published by Dove Medical Press Limited, and licensed under Creative Commons Attribution - Non Commercial (unported, v3.0)

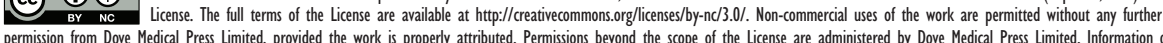
permission for how to request permission may be found at: http:///www.dovepress.com/permissions.php 
Randomized Protocol [SHARP] and Sorafenib Asia-Pacific trial). ${ }^{1,3}$ Sorafenib is a multikinase inhibitor; it inhibits angiogenesis by targeting vascular endothelial growth factor receptor 2 and platelet-derived growth factor receptor pathways while also blocking cell proliferation by targeting the Ras/mitogen-activated protein kinase signaling pathway. ${ }^{5}$ Although sorafenib has been shown to improve median overall survival (OS) outcomes in patients with advanced HCC, the median OS in these patients remains $<1$ year. $^{6}$

In addition to systemic therapy with sorafenib for unresectable HCC, radioembolization with yttrium-90 resin microspheres (Y90 RMS) is a recognized liver-directed therapy whose role is still being refined owing to a lack of randomized trials. ${ }^{7}$ In radioembolization, implantable radioactive microspheres are delivered into the arteries that feed the tumors. The high-energy Y90 radiation source emits a tumoricidal dose of beta radiation with a mean tissue penetration of $2.5 \mathrm{~mm}$ and a maximum tissue penetration of $11 \mathrm{~mm}$, limiting radiation exposure in the surrounding normal parenchyma. ${ }^{8}$ Treatment with Y90 RMS has been found to reduce tumor burden and may help downstage the patient for radical therapies. Y90 RMS can be administered in the presence of portal vein thrombosis and in cirrhosis patients with good liver function. ${ }^{7}$ Sinusoidal obstruction syndrome is the main complication in patients with a noncirrhotic liver. ${ }^{9}$

At our institution, we introduced treatment with Y90 RMS to patients with advanced HCC who were receiving standard or reduced doses of sorafenib. The purpose of this combination therapy was to optimize local control of the disease with systemic and liver-directed therapies. Given that the role of Y90 RMS combined with sorafenib in advanced HCC remains unclear, in this retrospective analysis, we studied the incidence of adverse events and survival outcomes with this combination therapy.

\section{Materials and methods}

\section{Study population and inclusion criteria}

We performed a single-center retrospective analysis of the incidence of adverse events, OS, progression-free survival (PFS), and hepatic or extrahepatic disease PFS following treatment with Y90 RMS in patients with advanced HCC who received systemic therapy with standard or reduced doses of sorafenib at our institution between January 2008 and May 2010. MD Anderson Cancer Center Institutional Review Board approval was obtained to review the medical records of the patients. The study was approved by the MD Anderson Cancer Center Institutional Review Board, who waived the need for informed patient consent as this was a retrospective review and no identifiable patient information is included in the manuscript.

A total of 19 patients fit our inclusion criteria, with a total of 21 RMS sessions performed. Two patients received a second treatment after initial responses. Patients with prior therapy were not excluded. For all patients, a multidisciplinary conference of treating physicians was held, and Y90 RMS was determined to be the best available treatment option for the patient at that particular stage of the disease. All patients were older than 18 years with unresectable HCC with or without portal vein invasion or extrahepatic disease. Patients had Barcelona Clinic Liver Cancer (BCLC) class B or C disease with a Child-Pugh score between A5 and B7 and Eastern Cooperative Oncology Group performance status of 0 or 1 . Five patients had concurrent or prior malignancies. All patients included in our analysis were followed up until April 2013 or death.

\section{Procedure and follow-up for treatment with Y90 RMS}

Prior to treatment with Y90 RMS, a computed tomography scan or magnetic resonance imaging was performed in all patients to evaluate tumoral and nontumoral volumes of the liver, portal vein patency, and extrahepatic disease. Liver and renal function tests were performed to confirm that they were within acceptable limits, including serum albumin $\geq 2.5 \mathrm{~g} /$ $\mathrm{dL}$, bilirubin $\leq 2 \mathrm{~g} / \mathrm{dL}$, and alanine aminotransferase and aspartate aminotransferase less than five times the normal values. Pretreatment planning diagnostic angiography was also performed in all patients to: 1) identify variant hepatic anatomy to confirm that it was adequate for treatment with Y90 RMS; 2) identify and embolize arteries supplying extrahepatic structures (eg, stomach and small bowel); 3) identify and embolize extrahepatic arterial supply of the tumor; 4) identify abnormal arteriovenous shunting; 5) evaluate portal venous status; and 6) administer Tc99m-labeled microaggregated albumin particles, which were used to determine the intrahepatic distribution of radiation particles and ensure that extrahepatic distribution did not occur as well as to determine the hepatopulmonary shunt fraction and radiation dose to the lungs. Patients with a hepatopulmonary shunt fraction of $\geq 20 \%$ were not given treatment with Y90 RMS and thus were not included in our analysis. Radiation doses (GBq) were calculated on the basis of body surface area $\left(\mathrm{m}^{2}\right)$ and percentage of tumor involvement in the liver: body surface area $-0.2+$ (tumor volume/liver volume).

After pretreatment imaging and angiography, the Y90 RMS treatment procedure was planned. Sorafenib was discontinued 1 week before the treatment and resumed 1 week 
after the procedure. Y90 RMS was injected through a microcatheter placed at a location determined by the angiogram. After the procedure, imaging of the bremsstrahlung was performed using planner and single-photon emission computed tomography imaging. Laboratory investigations were performed 1 week after the procedure and then every 4 weeks or as needed. Follow-up computed tomography scans of the abdomen were obtained at 8- to 12 -week intervals.

\section{Retrospective data collection and analysis}

Patient charts were reviewed, and the following data were collected: demographic information, possible etiology of HCC, imaging appearance of the tumor, extent and location of the extrahepatic disease, laboratory data before and after the Y90 RMS procedure, dosage and duration of treatment with sorafenib before and after treatment with Y90 RMS, Y90 RMS treatment details, Response Evaluation Criteria in Solid Tumors (RECIST) and European Association for the Study of the Liver (EASL) responses according to imaging, other concurrent therapies given, adverse events occurring after treatment with Y90 RMS according to Common Terminology Criteria for Adverse Events (CTCAE), sorafenib-related toxic effects, and OS duration, which was defined as the time interval between the start of the treatment with sorafenib and the date of death or last follow-up. The data obtained were also analyzed to determine the PFS duration for each patient, which was defined as the time interval between the day of initiation of sorafenib and the date of disease progression or death. We also determined PFS specifically for hepatic disease and extrahepatic disease.

OS, PFS, and response rate (according to RECIST criteria) were compared between those with BCLC class B disease and those with BCLC class C disease. Univariate analysis was performed using the chi-square test or Fisher's exact test for categorical variables and the Kruskal-Wallis test for continuous variables. The Kaplan-Meier method was used to estimate median OS and PFS values, and the log-rank test was used to detect statistically significant differences in survival between those with BCLC class B disease and those with BCLC class $C$ disease. For all statistical analyses, a two-sided $P$-value was used as a descriptive measure; $P<0.05$ was considered statistically significant. We used IBM SPSS Statistics for Windows, Version 21.0 (IBM Corporation, Armonk, NY, USA) for all data management and statistical analysis.

\section{Results}

\section{Patient characteristics}

Among the 19 patients whose records were reviewed in our study, $14(74 \%)$ were men. The median age at the time of treatment with Y90 RMS and sorafenib was 67 years (range: 51-82 years). Demographic and clinicopathologic characteristics of the patients are shown in Table 1.

The median follow-up, defined as the time between the start of the treatment with sorafenib and death or the last available follow-up, was 19.3 months (95\% confidence interval [CI], 9.6-33.7 months). At the time of our analysis (April 2013), five patients were alive and 14 had died. The median follow-up time in patients who had died was 15.7 months (95\% CI, 9.2-23.1 months) and in patients who were alive was 35.5 months (95\% CI, 9.6-40.4 months).

\section{Treatment details}

Patients received treatment with sorafenib for a median of 224 days (49-732 days). Prior to treatment with Y90

Table I Demographic characteristics, risk factors, and clinicopathologic characteristics of HCC patients treated with sorafenib + Y90 $(n=19)$

\begin{tabular}{|c|c|c|c|}
\hline Variable & $n=19$ & $\%$ & $95 \% \mathrm{Cl}$ \\
\hline \multicolumn{4}{|l|}{$\overline{S e x}$} \\
\hline Male & 14 & 74 & $0.49-0.91$ \\
\hline Female & 5 & 26 & $0.09-0.51$ \\
\hline \multicolumn{4}{|l|}{ Risk factors } \\
\hline Diabetes & 7 & 37 & $0.16-0.62$ \\
\hline HBV & 4 & 21 & $0.06-0.46$ \\
\hline $\mathrm{HCV}$ & 3 & 16 & $0.03-0.4$ \\
\hline Alcohol abuse & 2 & II & $0.01-0.33$ \\
\hline $\mathrm{NASH}$ & 1 & 5 & $0.00 I-0.26$ \\
\hline Adenoma & I & 5 & $0.00 I-0.26$ \\
\hline Unknown & 3 & 16 & $0.03-0.4$ \\
\hline Concurrent malignancies $^{\mathrm{a}}$ & 3 & 16 & $0.03-0.4$ \\
\hline Prior malignancies ${ }^{\mathrm{b}}$ & 2 & 11 & $0.01-0.33$ \\
\hline Extrahepatic disease & 7 & 37 & $0.16-0.62$ \\
\hline PVT & 7 & 37 & $0.16-0.62$ \\
\hline HVT & 2 & 11 & $0.01-0.33$ \\
\hline Portal hypertension & 5 & 26 & $0.09-0.51$ \\
\hline \multicolumn{4}{|l|}{ AFP levels at diagnosis } \\
\hline$>400 \mathrm{ng} / \mathrm{mL}$ & 6 & 32 & $0.13-0.57$ \\
\hline$<400 \mathrm{ng} / \mathrm{mL}$ & 13 & 68 & $0.43-0.87$ \\
\hline \multicolumn{4}{|l|}{ ECOG } \\
\hline 0 & 6 & 32 & $0.13-0.57$ \\
\hline । & 13 & 68 & $0.43-0.87$ \\
\hline \multicolumn{4}{|l|}{ CTP } \\
\hline$A$ & 16 & 84 & $0.6-0.97$ \\
\hline B & 3 & 16 & $0.03-0.4$ \\
\hline \multicolumn{4}{|l|}{$\mathrm{BCLC}$} \\
\hline B & 6 & 32 & $0.13-0.57$ \\
\hline C & 13 & 68 & $0.43-0.87$ \\
\hline
\end{tabular}

Notes: aConcurrent malignancies included renal cell cancer, multiple myeloma, and prostate cancer; 'prior malignancies included lymphoma and bladder cancer.

Abbreviations: $\mathrm{HCC}$, hepatocellular carcinoma; $\mathrm{Cl}$, confidence interval; $\mathrm{HBV}$, hepatitis B virus; HCV, hepatitis C virus; $\mathrm{NASH}$, nonalcoholic steatohepatitis; PVT, portal vein thrombosis; HVT, hepatic vein thrombosis; AFP, alfa feto protein; ECOG, Eastern Cooperative Oncology Group; CTP, Child-Turcotte-Pugh score; BCLC, Barcelona Clinic Liver Cancer. 
RMS, patients received sorafenib for a median of 58 days (26-732 days) and after treatment with Y90 RMS, 140 days (33-475 days). Nine patients tolerated the full dose of sorafenib, and ten patients received a reduced dose owing to toxic effects prior to treatment with Y90 RMS. Prior to treatment with sorafenib, four patients received systemic therapy with bevacizumab, erlotinib, capecitabine, or ADI PEG 20. After treatment with Y90 RMS, nine patients received various locoregional therapies, including transarterial chemoembolization with drug-eluting beads, Y90 glass microspheres, or hepatic arterial infusion therapy, or systemic therapy with bevacizumab, erlotinib, and/or capecitabine. Whole-liver Y90 RMS was administered in 12 patients, and lobar Y90 RMS was administered in seven patients. Two patients received two whole-liver treatments after the initial response. The median hepatopulmonary shunt fraction was $9.8 \%(3.6 \%-19.9 \%)$, and the median radiation activity delivered was $41.2 \mathrm{mCi}(29.6-65.2 \mathrm{mCi})$.

\section{Adverse events}

Ninety days after the treatment with Y90 RMS, no CTCAE grade IV adverse events were noted. However, one patient (5\%) had a grade I adverse event, five patients (26\%) had grade II adverse events, and four patients (21\%) had grade III adverse events, including gastrointestinal ulcer, mucositis, lymphopenia without life-threatening infection, and abdominal pain or nausea and vomiting.

\section{Response analysis}

As per RECIST criteria, 90 days after treatment with Y90 RMS, a stable disease was found in 17 of the 21 patients (81\%) and partial response was found in four patients (19\%); none of the patients reported a complete response. According to EASL criteria, 90 days after treatment with Y90 RMS, stable disease was found in 13 patients $(62 \%)$ and partial response was found in eight patients $(38 \%)$; none of the patients reported a complete response (Table 2).

\section{Survival outcomes}

The survival outcomes included OS, overall PFS, hepatic disease PFS, and extrahepatic disease PFS for all patients and for subgroups with BCLC class B and C disease. Patients with BCLC class B disease had significantly better OS and extrahepatic disease PFS outcomes than those with BCLC class $\mathrm{C}$ disease (Table 2 and Figure 1).

\section{Discussion}

Because more than $80 \%$ of patients with HCC are not candidates for curative treatments, such as resection or liver transplantation, patient outcomes remain very poor. Recently, sorafenib was found to improve survival in patients with unresectable HCC and Child-Pugh class A disease. In the SHARP trial, the median OS was 10.7 months for patients treated with sorafenib compared with 7.9 months for those receiving a placebo $(P=0.0006)$, and the median time to progression was 5.5 months for those treated with sorafenib compared with 2.8 months for those receiving a placebo $(P<0.001){ }^{1}$ In our study of patients treated with both sorafenib and Y 90 RMS, survival outcomes were substantially higher, median OS was 19.52 months, and median PFS was 6.63 months. These data suggest that treatment by adding Y90 RMS to sorafenib may improve survival outcomes over treatment with sorafenib alone in patients with advanced HCC. Moreover, the combination of treatment with Y90 RMS and sorafenib was well tolerated; our data showed that the incidence of adverse events was low.

Notably, patients in the SHARP trial with extrahepatic spread or macrovascular invasion were found to have poorer survival outcomes than those who did not have these conditions. ${ }^{1}$ Considering that the most common cause of HCC-related death in patients with extrahepatic spread is progression of intrahepatic HCC causing hepatic failure rather than progression of extrahepatic metastasis, the addition of an intra-arterial local therapy, such as Y90 RMS, could improve survival outcomes and quality of life in these patients.

Overall, sorafenib has been found to be well tolerated; in the SHARP trial, diarrhea and hand-foot skin reactions were the most commonly reported adverse events $(8 \%$ of patients). ${ }^{1}$ Sorafenib inhibits molecular components of the Raf-MEK-ERK signaling pathway, leading to containment of tumor growth and inhibition of vascular endothelial growth factors 1,2 , and 3 and platelet-derived growth factor beta. ${ }^{5}$

Table 2 Survival outcomes of all patients in our study $(n=19)$ and patients subcategorized by BCLC stage (class B: $n=6$; class C: $\mathrm{n}=13)$

\begin{tabular}{|c|c|c|c|c|}
\hline BCLC Stage & $\begin{array}{l}\text { Median overall survival, } \\
\text { months }(95 \% \mathrm{Cl})\end{array}$ & $\begin{array}{l}\text { Median hepatic disease } \\
\text { PFS, months }(95 \% \mathrm{Cl})\end{array}$ & $\begin{array}{l}\text { Median extrahepatic disease } \\
\text { PFS, months }(95 \% \mathrm{Cl})\end{array}$ & $\begin{array}{l}\text { PFS, months } \\
(95 \% \mathrm{Cl})\end{array}$ \\
\hline All patients & $19.52(9.23-33.68)$ & $7.82(6.37-10.87)$ & $8.94(6.64-33.68)$ & $6.63(5.49-7.82)$ \\
\hline BCLC class B & $54.97(8.38-54.97)$ & $9.3(6.28-32.1)$ & $38.01(5.49-54.97)$ & $7.18(5.49-32.1)$ \\
\hline BCLC class $\mathrm{C}$ & $12.12(8.94-23.06)$ & $6.97(6.14-12.12)$ & $7.52(5.09-\mid 1.47)$ & $6.64(4.9-7.52)$ \\
\hline
\end{tabular}

Abbreviations: BCLC, Barcelona Clinic Liver Cancer; $\mathrm{Cl}$, confidence interval; PFS, progression-free survival. 

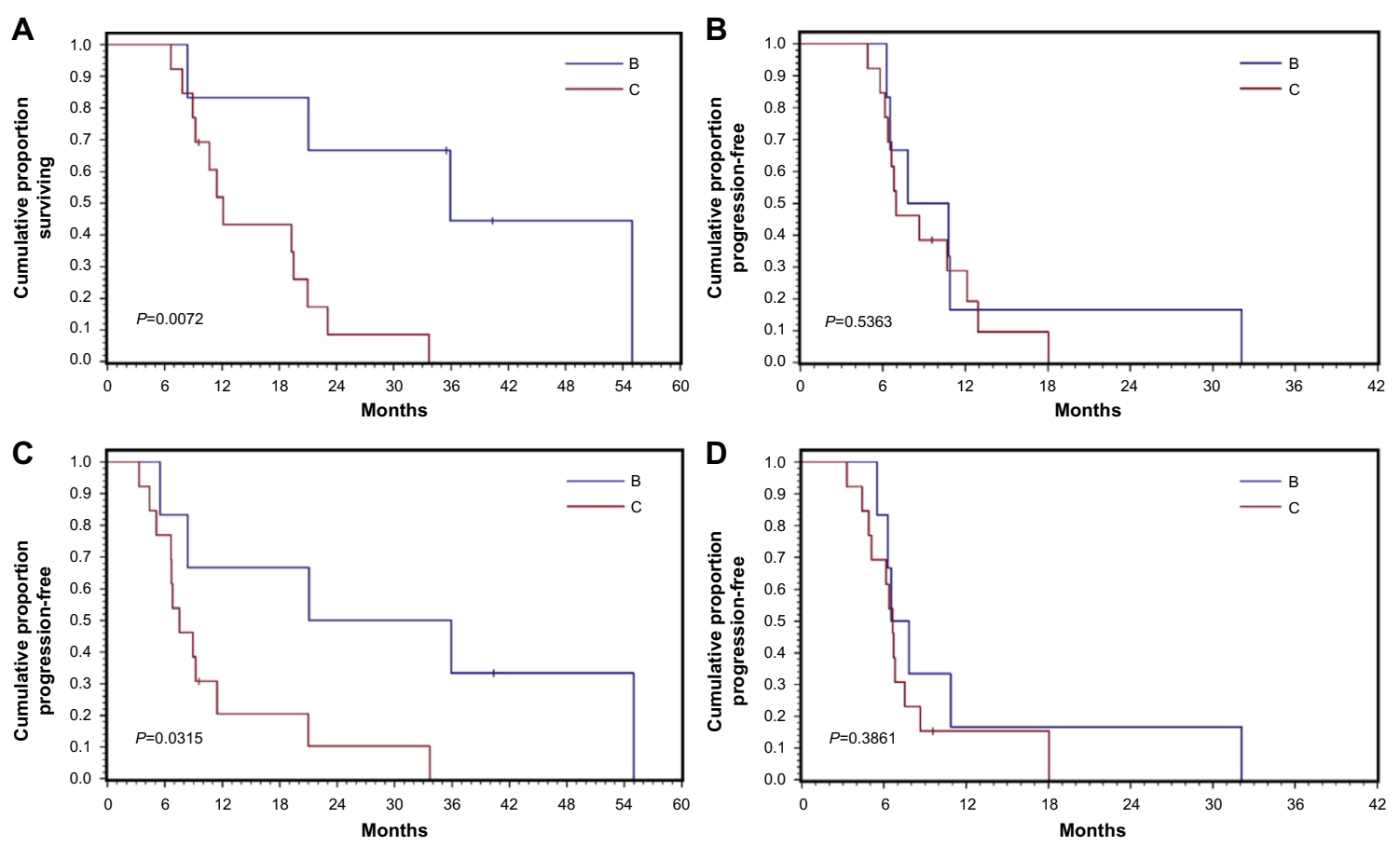

Figure I Kaplan-Meier survival curves for patients with BCLC class B and class C diseases: (A) overall survival, (B) hepatic disease progression-free survival, (C) extrahepatic disease progression-free survival, and (D) progression-free survival.

Abbreviation: BCLC, Barcelona Clinic Liver Cancer.

Thus, by inhibiting tumor growth and neoangiogenesis, sorafenib targets two key pathways that lead to growth of HCC. However, despite encouraging reports from clinical trials of sorafenib for the treatment of HCC, the median OS in patients with advanced $\mathrm{HCC}$ remains $<1$ year. Several ongoing clinical trials are studying the combination of sorafenib with locoregional therapies, such as chemoembolization, ${ }^{10,11}$ radiotherapy, ${ }^{12}$ and radioembolization with $\mathrm{Y} 90,{ }^{13,14}$ with the intent of identifying optimal combination therapies to improve survival outcomes in patients with advanced unresectable HCC. All patients in our retrospective analysis had unresectable HCC and a Child-Pugh score between A5 and B7, and they received reduced or full doses of sorafenib. The decision to offer treatment with Y90 RMS was made on a case-by-case basis by multidisciplinary consensus on the basis of the status of the patient's disease at that particular time. The concept of combining radiotherapy with antiangiogenic systemic chemotherapy to treat solid tumors has been studied previously in preclinical trials and was found to show synergistic antitumor activity, ${ }^{15}$ with prolongation of time to progression and improved local tumor control. Antiangiogenic agents reduce tumor hypoxia by normalizing tumor vascularity and by improving delivery of oxygen and therapeutic agents to the tumor, which increases the radiosensitivity of the tumor. Radiotherapy then activates the ceramide pathway, leading to apoptosis and cell death.
The combination of radiotherapy and agents targeting the vascular endothelial growth factor receptor has been shown to have synergistic activity against endothelial cells. ${ }^{16-18} \mathrm{Li}$ et $\mathrm{al}^{19}$ also showed that sorafenib combined with radiotherapy exerted a schedule-dependent effect on HCC cells in vitro. In that study, sorafenib administered after irradiation potentiated the tumor-inhibitory effect of the radiation.

We retrospectively evaluated the combination of Y90 RMS and sorafenib in patients with advanced HCC with the primary intent of evaluating the incidence of adverse events. Although the safety of both sorafenib and Y90 RMS has been well documented, the safety of concurrent use of these treatments is not known. Because of this, sorafenib was discontinued 1 week before Y90 RMS was administered and resumed 1 week after completion of the treatment in our patient population. The incidence of adverse events noted in patients receiving sorafenib alone in the SHARP trial was similar to that observed in our retrospective analysis. Ninety days after treatment with Y90 RMS, no patients developed CTCAE grade IV adverse events and only four patients developed CTCAE grade III adverse events. Although we reviewed a limited number of patients in our study, this finding is relevant because the safety of this combination therapy for human subjects has not been well reported. Prospective clinical trials with high target enrollment numbers are currently being conducted to more 
firmly establish the safety of this combination therapy. Once the safety of the approach is confirmed, a wide range of therapeutic combinations could be studied to identify the optimal approach and possibly increase survival in patients with advanced HCC.

We also studied the efficacy of the combination of Y90 RMS and sorafenib in our retrospective analysis. The median OS from the start of treatment with sorafenib was 19.52 months (95\% CI, 9.23-33.68 months), and OS rates were $62.3 \%$ at 1 year and $31.7 \%$ at 2 years, according to Kaplan-Meier analysis. These findings are impressive when compared with those of the SHARP trial (median OS, 10.7 months; 1-year OS rate, 44\%) ${ }^{1}$ and the Sorafenib AsiaPacific trial (median OS, 6.5 months). ${ }^{3}$ PFS in our study was 6.63 months, which is 1 month longer than that reported in the SHARP trial. We also observed a partial response in $19 \%$ of patients and stable disease in $81 \%$ of patients. This is superior to data reported in the SHARP trial, in which partial response was recorded in $2 \%$ of patients and stable disease in $71 \%$ of patients. ${ }^{1}$

We found a marked difference in the survival outcomes between those with BCLC class B disease and those with BCLC class $\mathrm{C}$ disease. The median OS in those with BCLC class B disease was 34.97 months compared with 12.12 months in those with BCLC class $\mathrm{C}$ disease $(P=0.007)$. The PFS was 7.18 months for BCLC class $\mathrm{B}$ disease and 6.64 months for BCLC class $\mathrm{C}$ disease. Although these differences were not statistically significant $(P=0.38)$, these findings suggest that patients with BCLC class B disease may respond better to the combination therapy than those with BCLC class C disease.

Although our findings are encouraging, our study contains some limitations. Our analysis included a small number of patients (21 sessions in 19 patients) and is retrospective in nature. Therefore, prospective, randomized controlled trials are warranted to evaluate the merit of our findings in patients with advanced HCC.

\section{Conclusion}

Our findings indicate that the combination of sorafenib and Y90 RMS for the treatment of advanced HCC is well tolerated; the addition of Y90 RMS to sorafenib did not appear to increase the incidence of adverse events compared with the treatment with sorafenib alone, which is reported in previous studies. Additionally, local response (partial response or stable disease) was achieved in all patients, and OS and PFS outcomes were superior to those reported in prior studies evaluating sorafenib alone in patients with a similar disease status. Future prospective clinical trials are warranted to confirm these findings and identify the best approach for this combination therapy.

\section{Acknowledgments}

This work was supported by the National Institutes of Health through grants CA170035-01 (to AOK) and CA106458-01 (to $\mathrm{MH}$ ). The contents of this article are solely the responsibility of the authors and do not necessarily represent the official views of the National Cancer Institute or the National Institutes of Health.

\section{Disclosure}

The authors report no conflicts of interest in this work.

\section{References}

1. Llovet JM, Ricci S, Mazzaferro V, et al. Sorafenib in advanced hepatocellular carcinoma. N Engl J Med. 2008;359(4):378-390.

2. Llovet JM, Bustamante J, Castells A, et al. Natural history of untreated nonsurgical hepatocellular carcinoma: rationale for the design and evaluation of therapeutic trials. Hepatology. 1999;29(1):62-67.

3. Cheng AL, Kang YK, Chen Z, et al. Efficacy and safety of sorafenib in patients in the Asia-Pacific region with advanced hepatocellular carcinoma: a phase III randomised, double-blind, placebo-controlled trial. Lancet Oncol. 2009;10(1):25-34.

4. Hall EJ. Radiobiology for the Radiologist. Philadelphia, PA: Lippincott Williams \& Wilkins; 2000.

5. Bruix J, Sherman M. Management of hepatocellular carcinoma: an update. Hepatology. 2011;53(3):1020-1022.

6. Narazaki M, Tosato G. Ligand-induced internalization selects use of common receptor neuropilin-1 by VEGF165 and semaphorin3A. Blood. 2006;107(10):3892-3901.

7. Sangro B, Carpanese L, Cianni R, et al. Survival after yttrium-90 resin microsphere radioembolization of hepatocellular carcinoma across Barcelona clinic liver cancer stages: a European evaluation. Hepatology. 2011;54(3):868-878.

8. Kennedy AS, Nutting C, Coldwell D, Gaiser J, Drachenberg C. Pathologic response and microdosimetry of $(90) \mathrm{Y}$ microspheres in man: review of four explanted whole livers. Int J Radiat Oncol Biol Phys. 2004;60(5):1552-1563.

9. Sangro B, Gil-Alzugaray B, Rodriguez J, et al. Liver disease induced by radioembolization of liver tumors: description and possible risk factors. Cancer. 2008;112(7):1538-1546.

10. Sacco R, Antonucci M, Bargellini I, Marceglia S, Mismas V, Cabibbo G. Transarterial chemoembolization and sorafenib in patients with intermediate-stage hepatocellular carcinoma: time to enter routine clinical practice? Future Oncol. 2015;11:1-3.

11. Yao X, Yan D, Liu D, Zeng H, Li H. Efficacy and adverse events of transcatheter arterial chemoembolization in combination with sorafenib in the treatment of unresectable hepatocellular carcinoma. Mol Clin Oncol. 2015;3(4):929-935.

12. Chen SW, Lin LC, Kuo YC, Liang JA, Kuo CC, Chiou JF. Phase 2 study of combined sorafenib and radiation therapy in patients with advanced hepatocellular carcinoma. Int J Radiat Oncol Biol Phys. 2014;88(5): 1041-1047.

13. Kulik L, Vouche M, Koppe S, et al. Prospective randomized pilot study of Y $90+/-$ sorafenib as bridge to transplantation in hepatocellular carcinoma. J Hepatol. 2014;61(2):309-317. 
14. Vouche M, Kulik L, Atassi R, et al. Radiological-pathological analysis of WHO, RECIST, EASL, mRECIST and DWI: imaging analysis from a prospective randomized trial of $990+/-$ sorafenib. Hepatology. 2013;58(5):1655-1666.

15. Ma BB, Bristow RG, Kim J, Siu LL. Combined-modality treatment of solid tumors using radiotherapy and molecular targeted agents. J Clin Oncol. 2003;21(14):2760-2776.

16. Abou-Alfa GK, Schwartz L, Ricci S, et al. Phase II study of sorafenib in patients with advanced hepatocellular carcinoma. J Clin Oncol. 2006;24(26):4293-4300.
17. Liu L, Cao Y, Chen C, et al. Sorafenib blocks the RAF/MEK/ERK pathway, inhibits tumor angiogenesis, and induces tumor cell apoptosis in hepatocellular carcinoma model PLC/PRF/5. Cancer Res. 2006;66(24):11851-11858.

18. Llovet JM, Burroughs A, Bruix J. Hepatocellular carcinoma. Lancet. 2003;362(9399):1907-1917.

19. Li Q, Hu Y, Xi M, He L, Zhao L, Liu M. Sorafenib modulates the radio sensitivity of hepatocellular carcinoma cells in vitro in a scheduledependent manner. BMC Cancer. 2012;12:485.

\section{Publish your work in this journal}

The Journal of Hepatocellular Carcinoma is an international, peerreviewed, open access journal that offers a platform for the dissemination and study of clinical, translational and basic research findings in this rapidly developing field. Development in areas including, but not limited to, epidemiology, vaccination, hepatitis therapy, pathology and molecular tumor classification and prognostication are all considered for publication. The manuscript management system is completely online and includes a very quick and fair peer-review system, which is all easy to use. Visit http://www.dovepress.com/testimonialsphp to read real quotes from published authors.

\footnotetext{
Submit your manuscript here: http://www.dovepress.com/journal-of-hepatocellular-carcinoma-journal
} 